\title{
Normal T2 map profile of the entire femoral cartilage using an angle/layer dependent approach
}

\author{
Yasuhito Kaneko, MD, PhD¹, Taiki Nozaki, MD", Hon Yu, PhD², Andrew Chang, MD1, \\ Kayleigh Kaneshiro, MD ${ }^{1}$, Ran Schwarzkopf, MD $^{3}$, Takeshi Hara, PhD $^{4}$, and Hiroshi \\ Yoshioka, MD, PhD ${ }^{1}$ \\ Yasuhito Kaneko: yasuhitk@uci.edu; Taiki Nozaki: nozakit@uci.edu; Hon Yu: hjyu@uci.edu; Andrew Chang: \\ andrelc1@uci.edu; Kayleigh Kaneshiro: kkaneshi@uci.edu; Ran Schwarzkopf: schwarzk@uci.edu; Takeshi Hara: \\ takeshi.hara@mac.com; Hiroshi Yoshioka: hiroshi@uci.edu \\ ${ }^{1}$ Department of Radiological Sciences, University of California, Irvine, Orange, California \\ 2John Tu and Thomas Yuen Center for Functional Onco-Imaging, University of California, Irvine, \\ Orange, California \\ ${ }^{3}$ Department of Orthopaedic Surgery, University of California, Irvine, Orange, California \\ ${ }^{4}$ Department of Intelligent Image Information, Division of Regeneration and Advanced Medical \\ Sciences, Gifu University Graduate School of Medicine, Gifu, Japan
}

\begin{abstract}
Purpose-To create standard T2 map profiles from the entire femoral cartilage of healthy volunteers in order to assess for regional variations using an angular and layer dependent approach.
\end{abstract}

Materials and Methods-Twenty healthy knees were evaluated using 3T sagittal images of a T2 mapping sequence. Manual segmentation of the entire femoral cartilage was performed slice by slice by two raters using Matlab. Inter- and intra-rater reliabilities were calculated using intraclass correlation coefficient (ICC) and Bland-Altman analysis. T2 values were analyzed with respect to specific locations (medial condyle, trochlea, and lateral condyle), angles to $\mathrm{B}_{0}$, and layers of cartilage (whole, deep, and superficial).

Results-Inter- and intra-rater reliability obtained from entire femoral cartilage was excellent (ICC $=0.84,0.86$, respectively). The ICCs around the trochlea were lower than those of the medial and lateral condyle. Both the inter- and intra-rater Bland-Altman plots indicated larger differences in pixel count are seen as the size of the angular segment becomes larger. T2 values were significantly higher in the superficial layer compared to the deep layer at each femoral compartment $(p<0.001)$. Magic angle effect was clearly observed, especially within the whole and deep layer over the medial and lateral femoral condyles, except for the superficial layer at the medial condyle.

Address correspondence and reprint request to: Hiroshi Yoshioka, MD, PhD, Department of Radiological Sciences, University of California, Irvine, 101 The City Drive South, Rt. 140, Orange, CA 92868, Phone: (714) 456-8849, FAX: (714)456-7430, hiroshi@uci.edu. 
Conclusion-The normal T2 map profiles of the entire femoral cartilage showed variations in ICCs by locations and in T2 values by angles and layers. These profiles can be useful for diagnosis of early cartilage degeneration in a specific angle and layer of each condyle and trochlea.

\section{Keywords}

MRI; T2 mapping; cartilage; femoral condyle; magic angle effect

\section{INTRODUCTION}

Osteoarthritis $(\mathrm{OA})$ is one of the most common chronic disorders worldwide, resulting in significant socioeconomic costs and morbidity (1). A number of therapeutic modalities have been developed for regenerative treatment of $\mathrm{OA}$, including chondroprotective and chondroregenerative drugs, osteochondral autografting, and autologous chondrocyte implantation (2-4). In order to effectively utilize these procedures, it is important to detect early cartilage degeneration and understand its natural progression.

Magnetic resonance imaging (MRI) is an ideal modality to assess early cartilage degeneration because of its ability to non-invasively quantify structural and biochemical changes associated with the onset and progression of OA (5-7). Novel MRI sequences for cartilage assessment, including T2 mapping, T1rho mapping, and delayed gadoliniumenhanced MR imaging of cartilage (dGEMRIC), have been developed as cartilage degeneration biomarkers for the evaluation of OA (8-15). T1rho mapping and dGEMRIC have been shown to be able to detect cartilage proteoglycan content depletion, which represents one of the earliest changes in cartilage degeneration $(8,9)$. T2 mapping is the most widely utilized technique for quantitative assessment of cartilage degeneration, and has been known to be sensitive to both the collagen matrix organization and water content of cartilage (16-18). The damaged collagen-proteoglycan matrix and increased water content in degenerating cartilage has reported to raise $\mathrm{T} 2$ value (12), which has been observed in patients with OA (10). Cartilage T2 value has been known to be dependent on location in the joint as well as layer (deep or superficial) of cartilage $(19,20)$. It is also known that the T2 value of cartilage is influenced by its orientation relative to the static magnetic field $\left(\mathrm{B}_{0}\right)$ due to the magic angle effect (21). Although many researchers have described T2 mapping of the knee cartilage in healthy populations, there are variations in the $\mathrm{T} 2$ values of healthy cartilage because of inconstant and varying definitions and limited number of subregions within the literatures $(19,22-32)$. Several studies have described the three-dimensional (3D) distribution of $\mathrm{T} 2$ values in the entire femoral cartilage $(33,34)$. However, these studies did not consider the effect of cartilage angle and layer dependency on $\mathrm{T} 2$ values.

The purpose of this study was to determine standard T2 map profiles of the entire femoral cartilage of young healthy volunteers with an angle and layer dependent approach. Further goals include establishing basic information regarding T2 mapping of the femoral condylar cartilage in the diagnosis of early knee OA.

J Magn Reson Imaging. Author manuscript; available in PMC 2016 December 01. 


\section{MATERIALS AND METHODS}

\section{Study population}

Twenty healthy volunteers (13 male and 7 female) without any history of knee pain or prior knee surgery were enrolled between January 2013 and June 2013. The mean age was 28.9 years (range, 19-38 years) and the mean weight measured $73 \mathrm{~kg}$ (range, 50-100 kg). The study protocol was approved by the institutional review board, and all volunteers gave written informed consent before any study-related procedures were done.

\section{Image acquisition}

One knee from each volunteer was scanned for an equal number of left/right knees using a 3-T MR scanner (Achieva, Philips Healthcare, Netherlands) with an 8-channel receive-only knee coil. There were no restrictions for all the subjects to perform daily activity and walk to the MRI suite before imaging. The imaging protocol consisted of a 2D, true sagittal to B0, turbo spin-echo T2 mapping sequence with the following acquisition parameters: repetition time/echo time, $2700 \mathrm{~ms} / 13,26,39,52,65,78$ and $91 \mathrm{~ms}$; bandwidth, $243 \mathrm{~Hz} /$ pixel; number of excitation, 1; field of view, $140 \times 140 \mathrm{~mm}$; slice thickness/gap, $3 \mathrm{~mm} / 0 \mathrm{~mm}$; flip angle, 90 degree; image matrix, $512 \times 512$; number of slices, 31 ; effective inplane spatial resolution, $0.27 \times 0.27 \mathrm{~mm}$; acceleration factor, 2.2; and total acquisition time, 13 minutes 26 seconds. Parallel imaging was used on all imaging sequences utilizing Sensitivity Encoding (SENSE) for MRI. Routine knee sequences including fast spin echo 2D fat suppressed (FS) sagittal proton density weighted images (PDWI), non-FS coronal PDWI, FS coronal PDWI, non-FS axial PDWI, and FS axial PDWI were also included in the protocol. This was done to confirm the normal appearance of each knee, as well as for use in future clinical applications.

\section{Image processing}

Cartilage segmentation of the entire femoral condyle-Manual cartilage extraction of the entire femoral condyle was performed on each $\mathrm{T} 2$ image by a board-certified orthopaedic surgeon (R1) and a board-certified radiologist sub-specialized in musculoskeletal radiology (R2) independently. For intra-rater reliability analysis, R1 performed a second cartilage segmentation for each subject at least 3 months after the first segmentation. Segmentation was performed on images with a TE value of $26 \mathrm{msec}$. This value was chosen due to the superior contrast between synovial fluid and cartilage, superior contrast between cartilage and the subchondral bone plate, and due to the higher signal to noise ratio compared to images from other TE values. Once the cartilage was segmented by manually placing vertices along the boundary, their $\mathrm{x}$ and $\mathrm{y}$ coordinates were used in a circle fitting algorithm by assuming a circular cartilage shape about an imaginary center position in the subchondral bone, whose coordinates were estimated in a least squares manner. We used the best fit center for each slice (Figure 1a). Additional boundary vertices with finer spacing were then interpolated and used for computation of slope angles for the radial vectors from the center position to each boundary vertex. For each slope angle (in step of 1degree) the farthest (closest to the articular surface) and nearest (closest to the bone) boundary vertices were recorded while the radial points between the boundary vertices were approximated by a linear interpolation and recorded for subsequent angle/layer-based 
segmentation of cartilage. T2 angle/layer-dependent profile was investigated in this study by angular segmentations in step of 4-degree over the length of the segmented cartilage (the angle 0 defined along $\mathrm{B}_{0}$ ), and by partitioning cartilage into deep (0-50\%) and superficial (51-100\%) layers (Figure 1b-e). All of the image processing described above was performed using an in-house developed and implemented software run in Matlab (Mathworks, Natick, MA).

\section{Normalization of slice numbers}

To define normalized slice numbers for each subject, we first reformatted coronal sections from sagittal images of knee MRI data using the software Medical Image Processing, Analysis and Visualization (MIPAV, Centre for Information Technology, National Institutes of Health, Bethesda, MD, USA), and defined the center of the medial femoral condyle (MC), lateral femoral condyle (LC), and femoral trochlea (T) in each subjects (Figure 2). We converted a total of 31 slices into 23 normalized slices for each subject, based on three anatomical landmarks for every 3-mm slice thickness, (e.g. MC+3, MC+6, T-3).

Normalization was necessary due to variations in knee size among the subjects. We choose to use 23 normalized slices because the mean transverse diameter of the 20 knees was $68.9 \mathrm{~mm}$. Figure 3 shows an overview of the image processing and normalization workflow.

\section{T2 values}

Once the cartilage boundaries were transferred onto the corresponding T2 maps, the segmented pixels with T2 values between 1.0 and $100 \mathrm{~ms}$ were used for the subsequent analyses to exclude the influence of outliers such as synovial fluid and chemical shift artifact (>100ms), and T2 values due to poor fit (0ms). We calculated the average T2 values at each normalized slice with respect to each angle and layer. Then, we created 2D-surface map of each cartilage layer. We also made 3D-graphs using a custom Matlab program where T2 profiles were shown as approximate curved surfaces. Additionally, we calculated the average $\mathrm{T} 2$ values at representative angles including $-90,-70,-54,-30,0,+30,+54,+70$, and +90 degrees for comparison of $\mathrm{T} 2$ values between magic angle and others.

\section{Statistical analysis}

Individual angular segments, each spanning 4 degrees over the length of the segmented cartilage, were used as the basis for all comparative tests. The inter- and intra-rater reliability of $\mathrm{T} 2$ values were assessed using intra-class correlation coefficient (ICC) analysis. ICC results of 0.60-0.74 were considered as "good", and ICC results higher than 0.74 as "excellent" (35). For the evaluation of the inter- and intra-rater reliability of the segmented area (number of pixels), Bland-Altman analysis was performed using only the overlapping data from the common slice/angular-ranges resulted from each test (36). Differences in cartilage T2 value between the deep and superficial layer at the center of each femoral compartment were assessed using unpaired $t$-test and Mann-Whitney test for normal and non-normal distributed data, respectively. T2 values between femoral compartments, and T2 values at different angles were compared using a Kruskal-Wallis test and Steel-Dwass post hoc test. Statistical analyses were performed using $\mathrm{R}$ version 3.0.2 for Windows software ( $\mathrm{R}$ Development Core Team, Vienna, Austria), except for the Bland-Altman analyses of 
segmented areas, which were calculated using Matlab. $P$ values less than 0.05 were considered to be statistically significant.

\section{RESULTS}

T2 relaxation time mapping for the entire femoral cartilage was successfully performed in all 20 healthy knees. There was no cartilage defect, meniscal damage, or ligament injury found in this study.

\section{Inter- and intra-rater reliability}

The ICCs in T2 value for inter- and intra-rater reliability are shown in Figure 4. The interand intra-rater ICCs of the entire femoral cartilage were either "good" or "excellent" for each layer (ICC $=0.62$ to 0.94 ). The ICCs with respect to each normalized slice showed that the inter- and intra-rater ICCs around the trochlea and both edges of the condyles were lower than those around the center of the medial and lateral condyle. The Bland-Altman analyses in segmented pixel number for inter- and intra-rater reliability are shown in Figure 5. The Bland-Altman plots showed that the size of angular segments from operator R1 was slightly larger on average than that of operator $\mathrm{R} 2$ (by $7 \pm 13.3$ (mean $\pm \mathrm{SD}$ ) pixels or $0.51 \pm 0.97$ $\mathrm{mm} 2$ for the whole layer). The mean difference in size was slightly smaller from the repeated segmentations by operator R1 (by $5 \pm 12.4$ pixels or $0.36 \pm 0.90 \mathrm{~mm} 2$ for the whole layer). Both the inter- and intra-rater Bland-Altman plots showed a statistically significant positive linear trend $(r=0.15 \& 0.20$, respectively) indicating larger differences in pixel count as the size of the angular segment became larger. Ninety-four percent of the inter-rater data points and $93 \%$ of the intra-rater data points were within $\pm 2 \mathrm{SD}$ of their respective linear fitted curve on the Bland-Altman plots.

\section{T2 values}

$\mathrm{T} 2$ values for the superficial and deep layers within the center of each of the three femoral compartments are shown in Table 1 . The average $\mathrm{T} 2$ value was significantly higher in the superficial layer than in the deep layer. The $\mathrm{T} 2$ value at the trochlea was significantly higher than those in each layer at the medial $(\mathrm{p}<0.01)$ and lateral condyle $(\mathrm{p}<0.05)$ except the superficial layer at the lateral condyle. There was no significant difference in the T2 value between the medial and lateral condyle in the whole and deep layer, but there was a significantly higher $\mathrm{T} 2$ value within the superficial layer at the lateral condyle compared to at the medial condyle $(\mathrm{p}<0.01)$. The $2 \mathrm{D}$ - and 3D-graphs showed inhomogeneous distribution of cartilage $\mathrm{T} 2$ values, and demonstrated magic angle effect clearly in each layer, especially within the deep layer of the medial and lateral condyle (Figure 6a-c, Figure $7 \mathrm{a}-\mathrm{c})$. Scatter plots of $\mathrm{T} 2$ values between magic angle and other representative angles demonstrate definite magic angle effect in each layer, especially within the whole and deep layer at $\pm 54^{\circ}$ over the medial and lateral femoral condyle, except for the superficial layer at the medial condyle (Figure 8a-f). 


\section{DISCUSSION}

\section{T2 mapping analysis of the entire femoral cartilage}

Most of the previous studies performed cartilage T2 mapping of the knee joint in the sagittal or coronal plane within a limited area of cartilage subregions (19, 22-32). Suroviec et al. reported significant differences in mean $\mathrm{T} 2$ values between 21 subregions in healthy volunteers (range: 41.7-54.2 ms), suggesting that there is a normal variation of T2 values within the knee (32). T2 values of the three anatomical landmarks in our study were similar to those of previous studies (range: $44.3-52.3 \mathrm{~ms})(27,32)$. Shiomi et al. reported the 3D distribution of $\mathrm{T} 2$ values of the entire femoral cartilage in healthy volunteer knees, in which cartilage $\mathrm{T} 2$ values for the internal half of the weight bearing zone covered by meniscus were significantly higher than those in all other zones within both the medial and lateral condyles (34). However, there has been no previous study that evaluated the cartilage T2 value of the entire femoral condyle with both angular orientation and cartilage layer taken into account. In the present study, we successfully evaluated the entire femoral cartilage T2 profile of healthy volunteer knees. Excellent inter- and intra-rater reliability in various locations from the medial to lateral condyle proved our angle and layer dependent approach for the entire femoral condyle is a useful method for cartilage T2 mapping analysis.

However, differences in reliability within specific locations suggest limitation or difficulty in our approach for specific areas such as the trochlea and far medial or lateral condyle. These findings may be due to the poor contrast between cartilage and surrounding tissue, partial volume effect or chemical shift artifact (37).

\section{T2 value and cartilage layer}

The mean T2 values of the superficial layer in our study were higher than those of the deep layer within each compartment, as reported in the previous study (22). The superficial layer in our approach contained the histological transitional layer. This layer is characterized by a more random orientation of the collagen fibers and increased water content compared with those in the deep layer (37), leading to a higher T2 value. Magic angle effect was more apparent in the deep layer compared to the superficial layer within both the medial and lateral condyles, which was the opposite outcome of previous studies $(22,28)$. Mosher et al. reported that the difference in cartilage $\mathrm{T} 2$ values between magic angle and $\mathrm{B}_{0}$ in healthy volunteers was greater in the superficial layer, yet smaller than those reported in the previous ex vivo studies, and concluded that regional differences in the degree of cartilage compression in weight bearing are primarily responsible for the regional differences in the cartilage T2 value (22). However, their results may be limited as they evaluated only a single sagittal slice of the lateral condyle. Our results were consistent with the histological fact that collagen fibers are perpendicular to subchondral bone in the deep layer, and are more susceptible to the magic angle effect, while the superficial layer has structural anisotropy of collagen fibers from the transitional zone to the superficial zone, and receives less effect of anisotropic orientation (37).

\section{T2 values and femoral compartment}

The T2 profile was clearly different between the medial and lateral condyles in this study. The medial condyle has a circular shape in the sagittal plane, and is more likely to exhibit 
the magic angle effect at $+54^{\circ}$ with smooth sinusoid curve. On the other hand, the lateral condyle has an oval or box-like shape, and showed a sharp and narrow peak at $+54^{\circ}$ in the deep layer in our image-processing manner using circle fitting algorithm. T2 values at the trochlea were higher than those of the medial and lateral condyle, as previously reported (32). The higher T2 value at the lateral condyle than the medial condyle in the superficial layer may be explained by the basis that the medial condyle has a wider weight bearing area than the lateral condyle (34), and that weight bearing areas have lower T2 values than nonweight bearing areas (38). Manual cartilage extraction was problematic at some locations. In particular, the worst inter- and intra-rater ICCs were seen around the trochlea (Figure 9a), where the cartilage-bone interface and cartilage surface were difficult to determine due to partial volume effect in $\mathrm{T} 2$ sagittal image (37).

\section{T2 values and angular orientation}

Most previous studies analyzed cartilage T2 values in comparison between subregions ( 25 , 32 ) or between weight bearing and non-weight bearing regions $(22,34)$. In this study, we analyzed the entire femoral cartilage in detail with in step of 4-degree. Angular orientation was seen to have a substantial effect on the T2 values within both condyles. Although the circular shape of the medial condyle in the sagittal plane was suitable for evaluating magic angle effect using our image-processing method, bimodal peaks of $\mathrm{T} 2$ value at $\pm 54^{\circ}$ were clearly observed over the entire medial and lateral condyle. However, there were no significant differences between the magic angle and other angles within the superficial layer of the medial condyle. This might be due to underestimation of the differences due to a small number of study subjects. Another potential reason could be that the structural anisotropy of the collagen fibers within the superficial layer decreased the magic angle effect (37). A large variation of the $\mathrm{T} 2$ value at $0^{\circ}$ in the superficial layer over the lateral condyle was probably due to a smoothly contoured thinning of the cartilage, the so-called "lateral femoral notch" (38), where it is easy to overestimate the cartilage thickness during manual extraction (Figure 9b).

Cartilage $\mathrm{T} 2$ values from this study demonstrated considerable variation with respect to each angular orientation and layer. These factors will have an impact during evaluation of subtle abnormalities of the femoral cartilage. The normal T2 profile of the entire femoral cartilage shown in this study can be a helpful benchmark in the diagnosis of early cartilage degeneration.

\section{Limitations}

Our study has several limitations. First, acquisition time was long. Acquisition times would need to be short in order to include T2 mapping sequences in clinical protocols. Shorter repetition time, shorter echo time, smaller matrix size, and limited slice number would contribute to a faster acquisition time (30,31), although these settings could make image quality poor. Second, post-processing, including manual segmentation of cartilage and normalization of slice number, was time-consuming. Automated or semi-automated segmentation and 2D/3D graph creation will likely become essential for shortening postprocessing time for future clinical applications. Third, the sample size of this study was small. We may need more samples to apply healthy T2 mapping profile for clinical atlas. 
We could have doubled the sample size by scanning both knees in each volunteer. Fourth, chemical shift artifact from a 3T magnet and the relatively narrow band width in this study might make discrimination between cartilage and subchondral bone difficult in some areas. Fifth, partial volume effect was inevitable with the 2D sequence in this study, and most prominent in the transitional area between the condyle and the trochlea, where the contour of the cartilage changes significantly (37). Sixth, we didn't assess other cartilage MRI techniques in this study such as dGEMERIC, which is known to be reliable for evaluating glycosaminoglycan content $(15,40)$, and T1rho, which is reported to be a more sensitive indicator of early cartilage degeneration than T2 mapping (41). The next step of our study will be an assessment of the femoral cartilage in early OA patients using T2 and T1rho techniques. Seventh, the intra-rater reliability wasn't assessed in both raters. In this study, we referred to previous studies regarding cartilage segmentation of the knee, in which the intra-rater reliability test was performed in a single rater $(34,42)$. Finally, we included only young healthy volunteers in this study. A large cohort of patients with early cartilage degeneration would need to be studied in order to prove our angle and layer dependent approach is clinically useful in the future.

In conclusion, this study demonstrated the standard T2 map profile from the entire femoral cartilage of normal volunteers with an angle/layer dependent approach. The inter- and intrarater reliability in $\mathrm{T} 2$ value and segmented pixel number was overall high with various agreements dependent on location. Variations in T2 value by angular orientation and cartilage layer was demonstrated throughout the femoral condyles. Our results can be useful for the diagnosis of early cartilage degeneration in a specific angle and layer of each condyle and trochlea by establishing a baseline normal $\mathrm{T} 2$ mapping profile.

\section{Acknowledgments}

Grant Support:

The work was funded by the National Center for Research Resources and the National Center for Advancing Translational Sciences, National Institutes of Health, through Grant UL1 TR000153.

\section{References}

1. Cleveland RJ, Luong ML, Knight JB, et al. Independent associations of socioeconomic factors with disability and pain in adults with knee osteoarthritis. BMC Musculoskelet Disord. 2013; 14:297. [PubMed: 24134116]

2. Behrens P, Bitter T, Kurz B, Russlies M. Matrix-associated autologous chondrocyte transplantation/ implantation (MACT/MACI):5-year follow-up. Knee. 2006; 13:194-202. [PubMed: 16632362]

3. Brittberg M, Lindahl A, Nilsson A, Ohlsson C, Isaksson O, Peterson L. Treatment of deep cartilage defects in the knee with autologous chondrocyte transplantation. N Engl J Med. 1994; 331:889-895. [PubMed: 8078550]

4. Goyal D, Keyhani S, Goyal A, Lee EH, Hui JH, Vaziri AS. Evidence-based status of osteochondral cylinder transfer techniques: a systematic review of level I and II studies. Arthroscopy. 2014; 4:497-505. [PubMed: 24680310]

5. Bashir A, Gray ML, Boutin RD, Burstein D. Glycosaminoglycan in articular cartilage: in vivo assessment with delayed Gd(DTPA)(2-)-enhanced MR imaging. Radiology. 1997; 205:551-558. [PubMed: 9356644] 
6. Mosher TJ, Dardzinski BJ, Smith MB. Human articular cartilage: influence of aging and early symptomatic degeneration on the spatial variation of T2-preliminary findings at $3 \mathrm{~T}$. Radiology. 2000; 214:259-266. [PubMed: 10644134]

7. Li X, Han ET, Ma CB, Link TM, Newitt DC, Majumdar S. In vivo 3T spiral imaging based multislice T(1rho) mapping of knee cartilage in osteoarthritis. Magn Reson Med. 2005; 54:929-936. [PubMed: 16155867]

8. Akella SV, Regatte RR, Gougoutas AJ, et al. Proteoglycan-induced changes in T1rho-relaxation of articular cartilage at 4T. Magn Reson Med. 2001; 46(3):419-423. [PubMed: 11550230]

9. Bashir A, Gray ML, Hartke J, Burstein D. Nondestructive imaging of human cartilage glycosaminoglycan concentration by MRI. Magn Reson Med. 1999; 41:857-865. [PubMed: 10332865]

10. Dunn TC, Lu Y, Jin H, Ries MD, Majumdar S. T2 relaxation time of cartilage at MR imaging: comparison with severity of knee osteoarthritis. Radiology. 2004; 232:592-598. [PubMed: 15215540]

11. Regatte RR, Akella SV, Lonner JH, Kneeland JB, Reddy R. T1rho relaxation mapping in human osteoarthritis (OA) cartilage: comparison of T1rho with T2. J Magn Reson Imaging. 2006; 23:547-553. [PubMed: 16523468]

12. Li X, Benjamin Ma C, Link TM, et al. In vivo $\mathrm{T}(1 \mathrm{rho})$ and $\mathrm{T}(2)$ mapping of articular cartilage in osteoarthritis of the knee using 3 T MRI. Osteoarthritis Cartilage. 2007; 15:789-797. [PubMed: 17307365]

13. Welsh GH, Trattnig S, Scheffler K, et al. Magnetization transfer contrast and T2 mapping in the evaluation of cartilage repair tissue with 3T MRI. J Magn Reson Imaging. 2008; 28:979-986. [PubMed: 18821633]

14. Koff MF, Parratte S, Amrami KK, Kaufman KR. Examiner repeatability of patellar cartilage T2 values. Magn Reson Imaging. 2009; 27:131-136. [PubMed: 18801631]

15. Watanabe A, Boesch C, Anderson SE, Brehm W, Mainil Varlet P. Ability of dGEMRIC and T2 mapping to evaluate cartilage repair after microfracture: a goat study. Osteoarthritis Cartilage. 2009; 17:1341e9. [PubMed: 19410029]

16. Nieminen MT, Rieppo J, Toyras J, et al. T2 relaxation reveals spatial collagen architecture in articular cartilage: a comparative quantitative MRI and polarized light microscopic study. Magn Reson Med. 2001; 46:487-493. [PubMed: 11550240]

17. Xia Y, Moody JB, Alhadlaq H. Orientational dependence of $\mathrm{T} 2$ relaxation in articular cartilage: a microscopic MRI (microMRI) study. Magn Reson Med. 2002; 48:460-469. [PubMed: 12210910]

18. Surowiec RK, Lucas EP, Ho CP. Quantitative MRI in the evaluation of articular cartilage health: reproducibility and variability with a focus on T2 mapping. Knee Surg Sports Traumatol Arthrosc. 2014; 22:1385-1395. [PubMed: 24170187]

19. Smith HE, Mosher TJ, Dardzinski BJ, et al. Spatial variation in cartilage T2 of the knee. J Magn Reson Imaging. 2001; 14:50-55. [PubMed: 11436214]

20. Goodwin DW, Wadghiri YZ, Zhu H, Vinton CJ, Smith ED, Dunn JF. Macroscopic structure of articular cartilage of the tibial plateau: influence of a characteristic matrix architecture on MRI appearance. AJR Am J Roentgenol. 2004; 182:311-318. [PubMed: 14736653]

21. Xia Y. Magic-angle effect in magnetic resonance imaging of articular cartilage: a review. Invest Radiol. 2000; 35:602-621. [PubMed: 11041155]

22. Mosher TJ, Smith H, Dardzinski BJ, Schmithorst VJ, Smith MB. MR imaging and T2 mapping of femoral cartilage: in vivo determination of the magic angle effect. AJR Am J Roentgenol. 2001; 177:665-669. [PubMed: 11517068]

23. Carballido-Gamio J, Stahl R, Blumenkrantz G, Romero A, Majumdar S, Link TM. Spatial analysis of magnetic resonance T1rho and T2 relaxation times improves classification between subjects with and without osteoarthritis. Med Phys. 2009; 36:4059-4067. [PubMed: 19810478]

24. Bae KT, Shim H, Tao C, et al. Intra- and inter-observer reproducibility of volume measurement of knee cartilage segmented from the OAI MR image set using a novel semi-automated segmentation method. Osteoarthritis Cartilage. 2009; 17:1589-1597. [PubMed: 19577672] 
25. Li X, Pai A, Blumenkrantz G, et al. Spatial distribution and relationship of T1rho and T2 relaxation times in knee cartilage with osteoarthritis. Magn Reson Med. 2009; 61:1310-1318. [PubMed: 19319904]

26. Stehling C, Lane NE, Nevitt MC, Lynch J, McCulloch CE, Link TM. Subjects with higher physical activity levels have more severe focal knee lesions diagnosed with 3T MRI: analysis of a nonsymptomatic cohort of the osteoarthritis initiative. Osteoarthritis Cartilage. 2010; 18:776-786. [PubMed: 20202488]

27. Mosher TJ, Liu Y, Torok CM. Functional cartilage MRI T2 mapping: evaluating the effect of age and training on knee cartilage response to running. 2010 Osteoarthritis Cartilage. 2010; 18:358364. [PubMed: 19948266]

28. Shiomi T, Nishii T, Myoui A, Yoshikawa H, Sugano N. Influence of Knee Positions on T2, T*2, and dGEMRIC Mapping in Porcine Knee Cartilage. Magn Reson Med. 2010; 64:707-714. [PubMed: 20535811]

29. Zarins ZA, Bolbos RI, Pialat JB, et al. Cartilage and meniscus assessment using T1rho and T2 measurements in healthy subjects and patients with osteoarthritis. Osteoarthritis Cartilage. 2010; 18:1408-1416. [PubMed: 20696262]

30. Mamisch TC, Trattnig S, Quirbach S, Marlovits S, White LM, Welsch GH. Quantitative T2 mapping of knee cartilage: differentiation of healthy control cartilage and cartilage repair tissue in the knee with unloading.initial results. Radiology. 2010; 254:818-826. [PubMed: 20123898]

31. Li X, Cheng J, Lin K, et al. Quantitative MRI using T1 $\rho$ and T2 in human osteoarthritic cartilage specimens: Correlation with biochemical measurements and histology. Magn Reson Imaging. 2011; 29:324-334. [PubMed: 21130590]

32. Surowiec RK, Lucas EP, Fitzcharles EK, et al. T2 values of articular cartilage in clinically relevant subregions of the asymptomatic knee. Knee Surg Sports Traumatol Arthrosc. 2014; 22:1404-1414. [PubMed: 24271329]

33. Akhtar S, Poh CL, Kitney RI. An MRI derived articular cartilage visualization framework. Osteoarthritis Cartilage. 2007; 15:1070-1085. [PubMed: 17707660]

34. Shiomi T, Nishii T, Nakata K, et al. Three-dimensional topographical variation of femoral cartilage T2 in healthy volunteer knees. Skeletal Radiol. 2013; 42:363-370. [PubMed: 23001118]

35. Oppo K, Leen E, Angerson WJ, Cooke TG, McArdle CS. Doppler perfusion index: an interobserver and intraobserver reproducibility study. Radiology. 1998; 208:453-457. [PubMed: 9680575]

36. Bland JM, Altman DG. Statistical methods for assessing agreement between two methods of clinical measurement. Lancet. 1986; 1:307-310. [PubMed: 2868172]

37. Waldschmidt JG, Rilling RJ, Kajdacsy-Balla AA, Boynton MD, Erikson SJ. In vitro and in vivo MR imaging of hyaline cartilage: zonal anatomy, imaging pitfalls, and pathologic conditions. Radiographics. 1997; 17:1387-1402. [PubMed: 9397453]

38. Stahl R, Blumenkrantz G, Carballido-Gamio J, et al. MRI-derived T2 relaxation times and cartilage morphometry of the tibio-femoral joint in subjects with and without osteoarthritis during a 1-year follow-up. Osteoarthritis Cartilage. 2007; 15:1225-1234. [PubMed: 17561417]

39. Yoshioka H, Stevens K, Genovese M, Dillingham MF, Lang P. Articular Cartilage of Knee: Normal Patterns at MR Imaging That Mimic Disease in Healthy Subjects and Patients with Osteoarthritis. Radiology. 2004; 231:31-38. [PubMed: 15068938]

40. Burstein D, Velyvis J, Scott KT, et al. Protocol Issues for Delayed Gd(DTPA)(2-)-Enhanced MRI (dGEMRIC) for Clinical Evaluation of Articular Cartilage. Magn Reson Med. 2001; 45:36-41. [PubMed: 11146483]

41. Choi JA, Gold GE. MR imaging of articular cartilage physiology. Magn Reson Imaging Clin N Am. 2011; 19(2):249-282. [PubMed: 21665090]

42. Fujinaga Y, Yoshioka H, Sakai T, et al. Quantitative Measurement of Femoral Condyle Cartilage in the Knee by MRI: Validation Study by Multireaders. J Magn Reson Imaging. 2014; 39:972977. [PubMed: 24123712] 

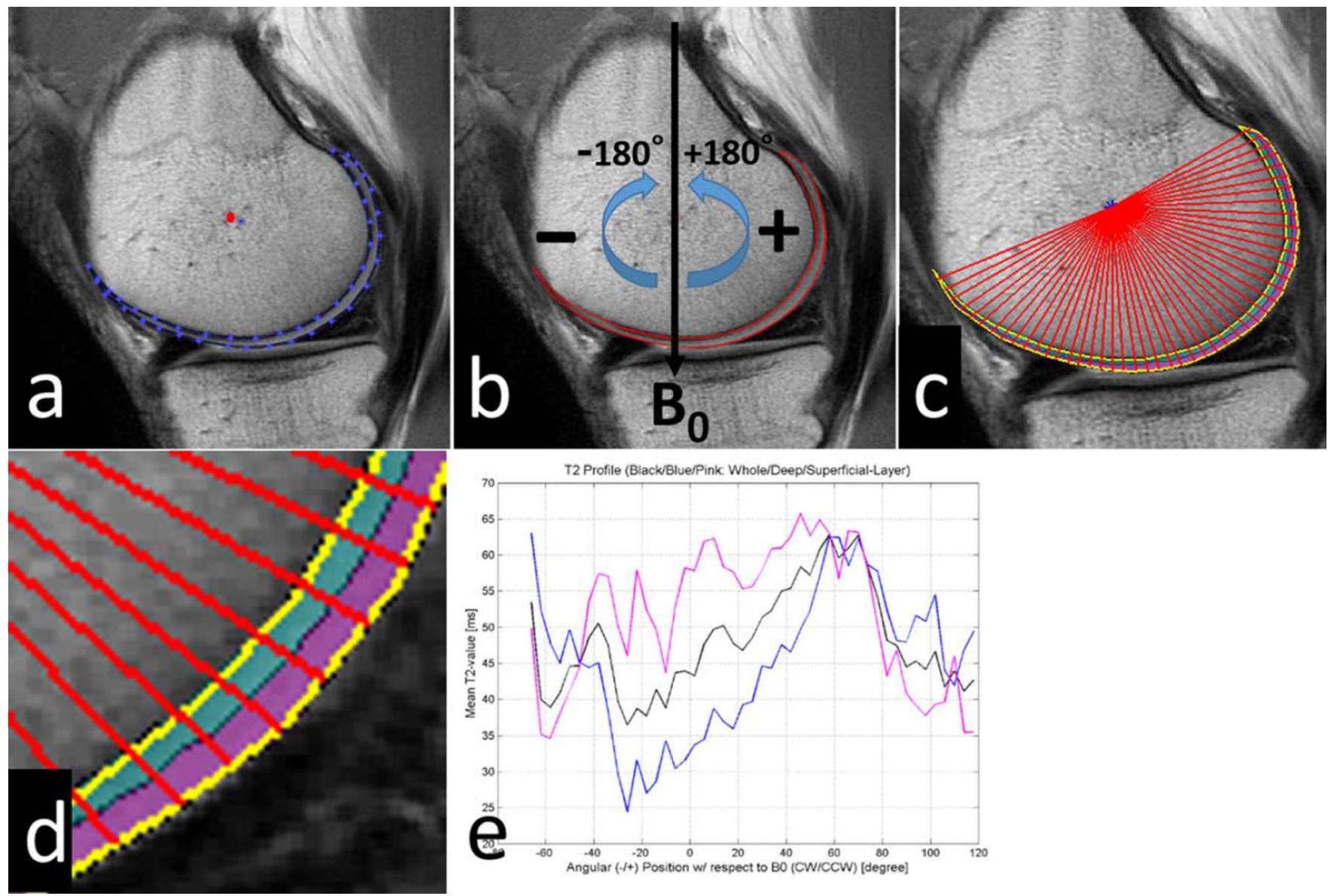

FIGURE 1.

Articular segmentation with angle/layer dependent approach. (a) After manual cartilage extraction, the central point of the cartilage (red dot) was automatically approximated. (b) Static magnetic field $\left(B_{0}\right)$ was defined as $0^{\circ}$, with negative/positive angles located anterior/ posterior to the central point. (c) Radial lines from a central point divided cartilage into 4degree segments. (d) Segmentation of cartilage into deep (0-50\%) and superficial layers (51-100\%) of relative thickness. (e) $T_{2}$ profiles were generated for whole thickness, deep, and superficial layers of cartilage. 

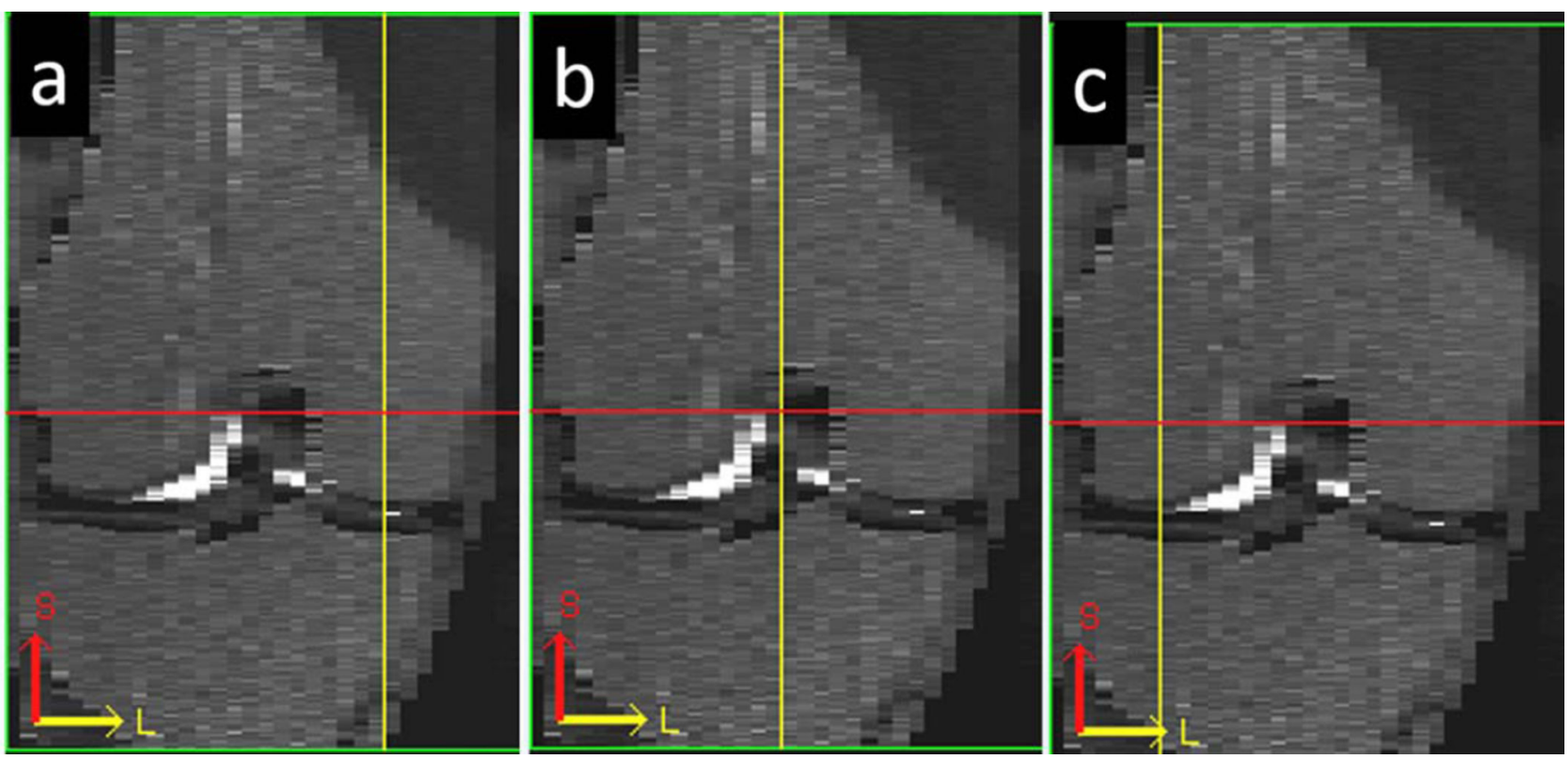

FIGURE 2.

Definition of three anatomical landmarks using reconstructed coronal images in MIPAV: (a) medial condyle, (b) trochlea, and (c) lateral condyle. 


\section{Journal of Magnetic Resonance Imaging}

\section{Total 31 sagittal slices}

$\frac{\Omega}{\frac{\pi}{\pi}}$

Manual cartilage extraction (Rater 1 and 2)

Automatic image processing (see Fig.1)

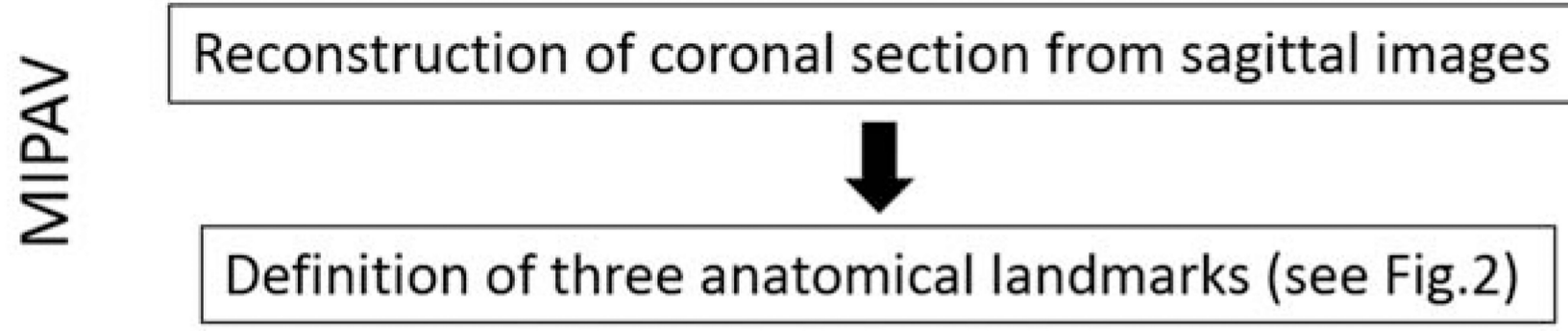

\section{3 normalized slices $(\mathrm{MC}+3, \mathrm{MC}+6, \mathrm{~T}-3,,$,}

FIGURE 3.

Flow diagram of image processing and normalization of slice numbers using MatLab and MIPAV. 


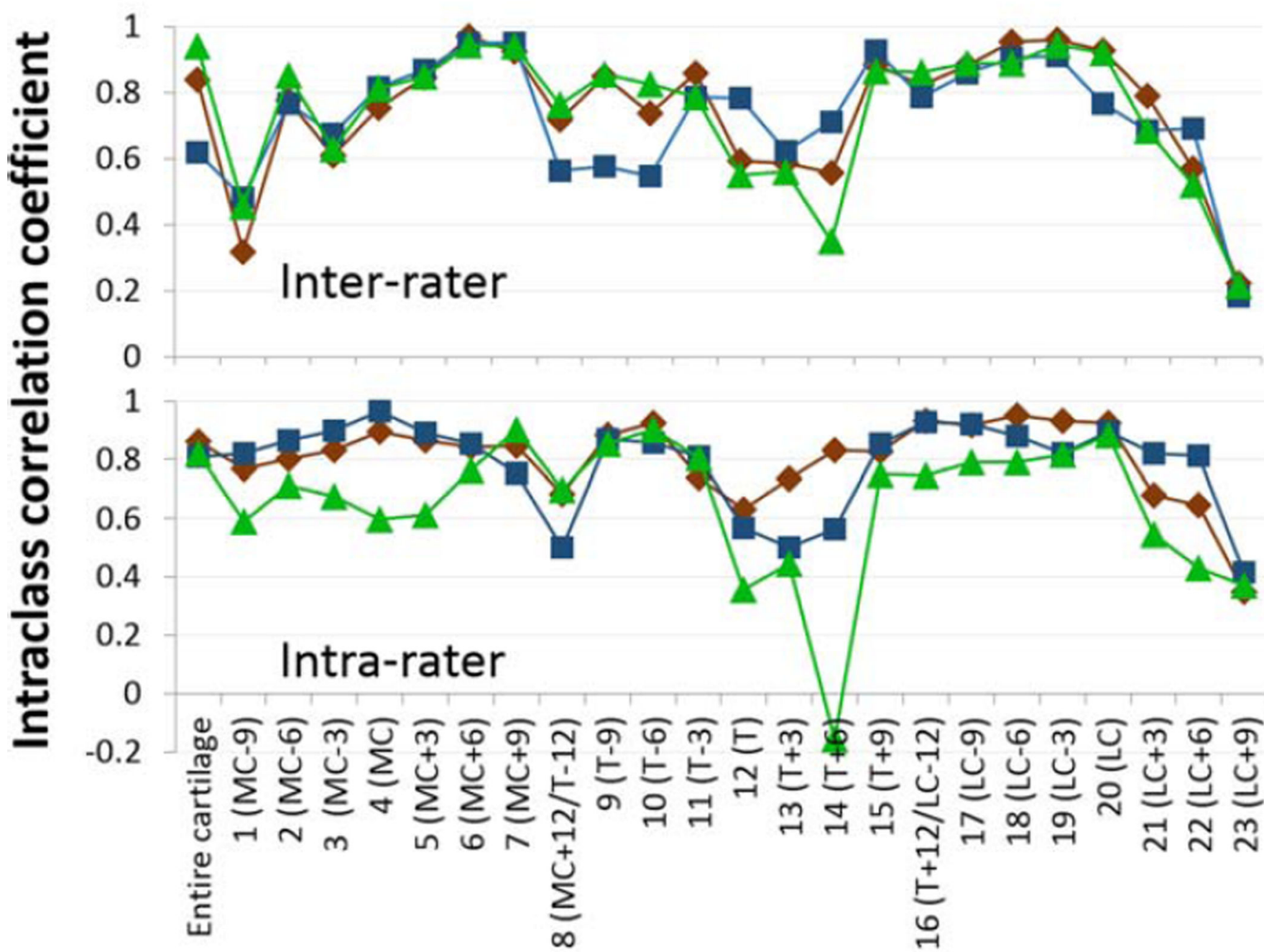

\section{Normalized slice number}

FIGURE 4.

Inter- and intrarater reliability of $T_{2}$ values in each layer of the cartilage with respect to each normalized slice number. MC, medial condyle; T, trochlea; LC, lateral condyle. Phrases shown in parentheses indicate the center of femoral compartment \pm distance $(\mathrm{mm})$. 
Inter-rater

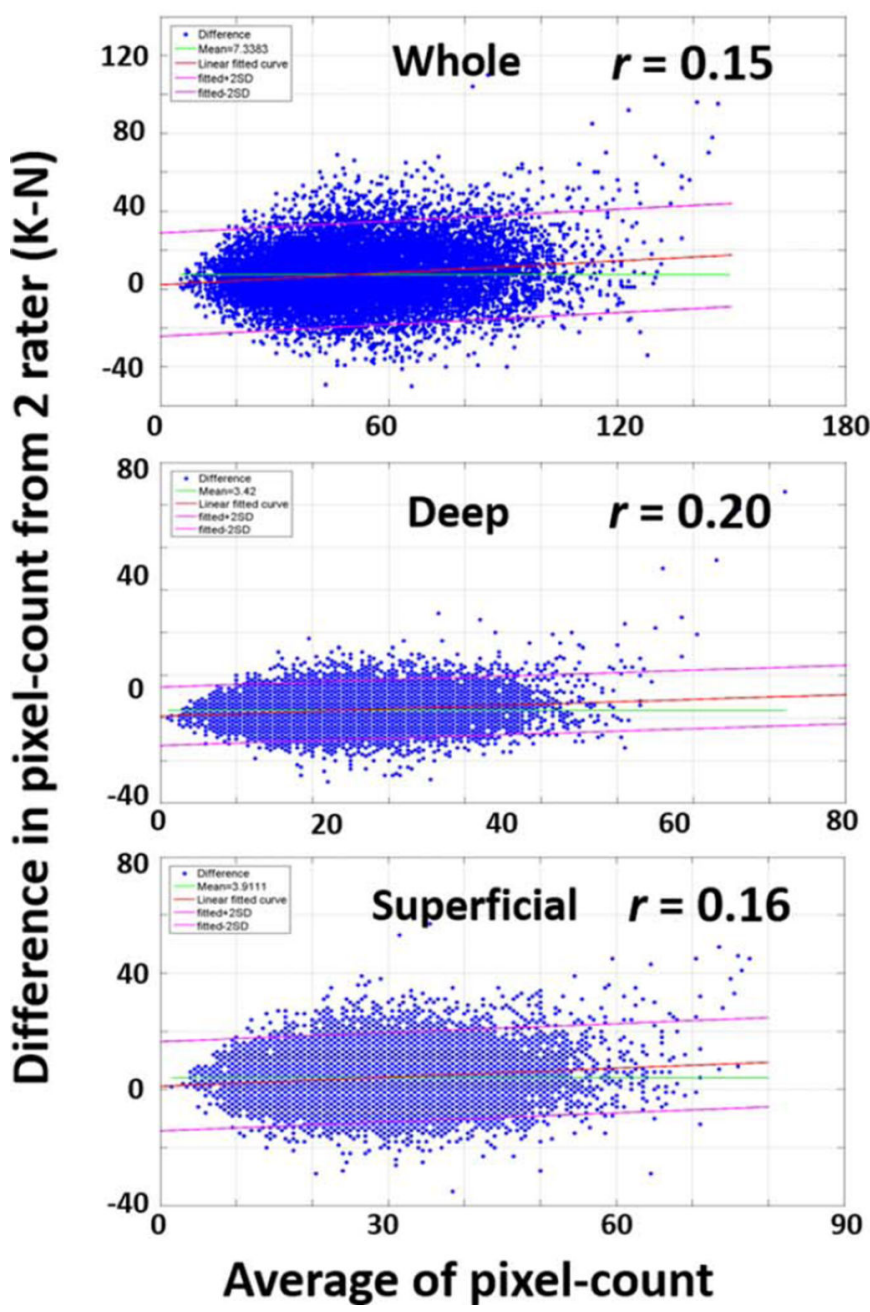

\section{Intra-rater}
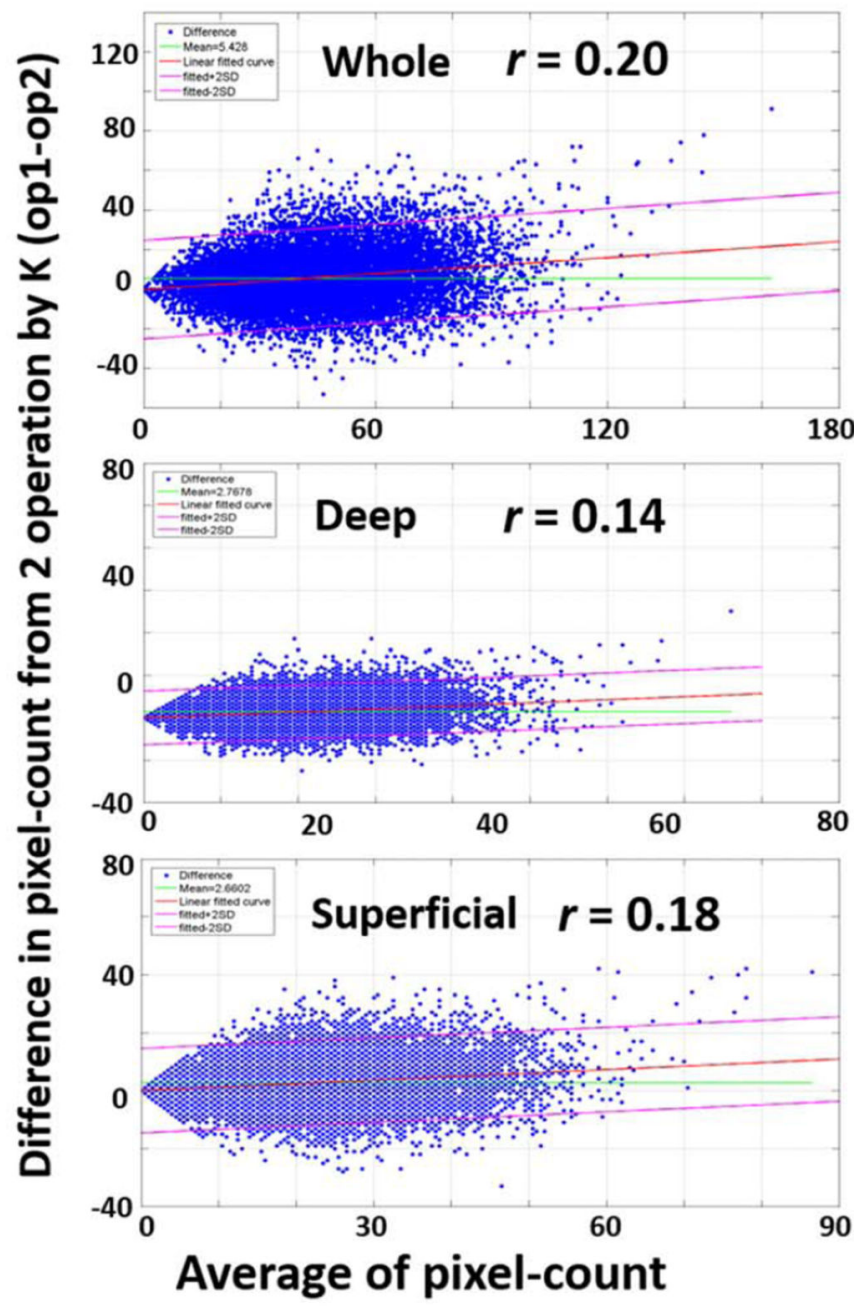

FIGURE 5.

Bland-Altman plots of inter- and intrarater agreements in segmented pixel number with respect to each layer of femoral cartilage. The green line, red line, and pink line indicate mean difference, linear fitted curve, and fitted $\pm 2 \mathrm{SD}$, respectively. 
(a) Whole

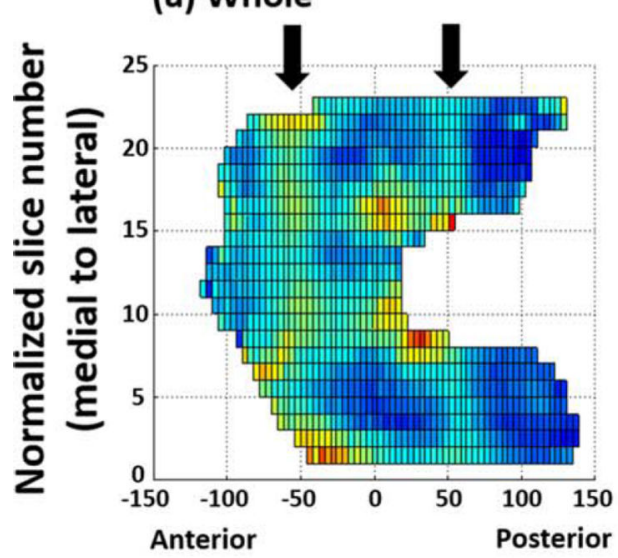

(b) Deep

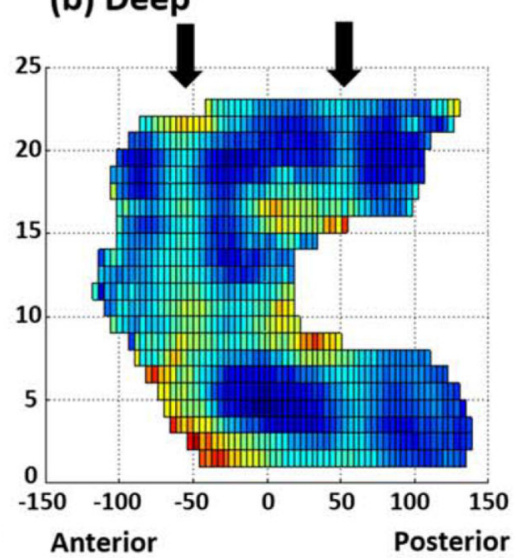

(c) Superficial

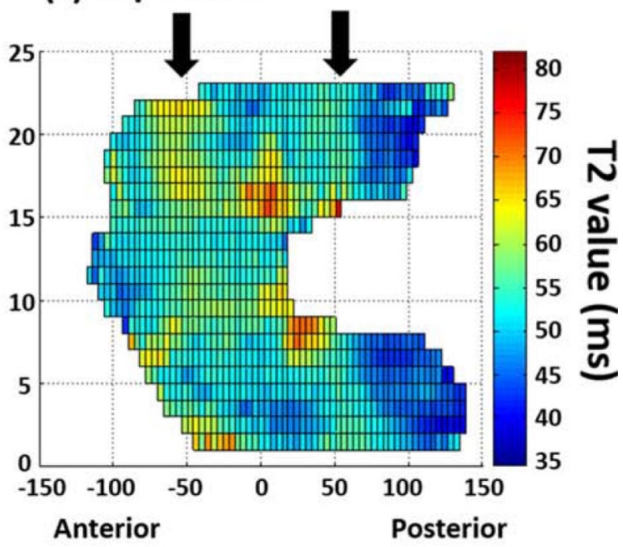

Angular orientation to $\mathrm{B}_{0}$ (degree)

FIGURE 6.

2D surface maps displaying the $T_{2}$ profile of: (a) the whole layer, (b) the deep layer, and (c) the superficial layer of the entire femoral cartilage. Arrows indicate $\pm 54.7^{\circ}$ (the magic angle). 
(a) Entire femoral cartilage (MC+T+LC)

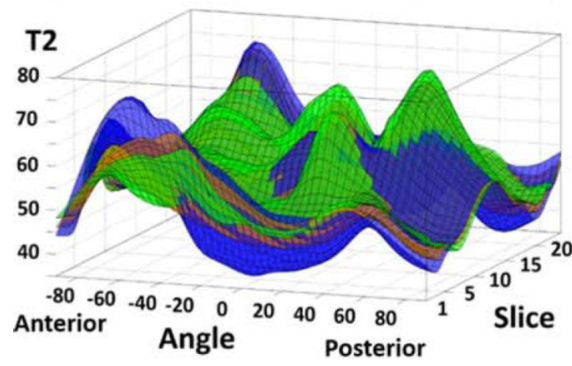

(b) Medial condyle

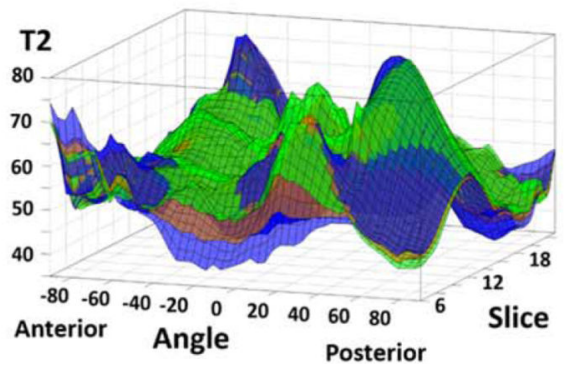

(c) Lateral condyle

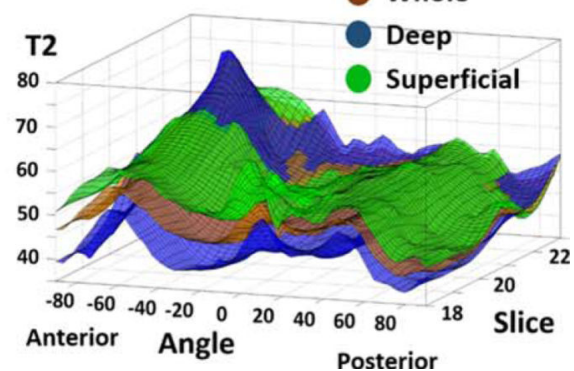

FIGURE 7.

3D graph of the $T_{2}$ profiles of: (a) the entire femoral cartilage, (b) cross-section at the center of the medial condyle, and (c) the lateral condyle. MC, medial condyle; T, trochlea; LC, lateral condyle. 

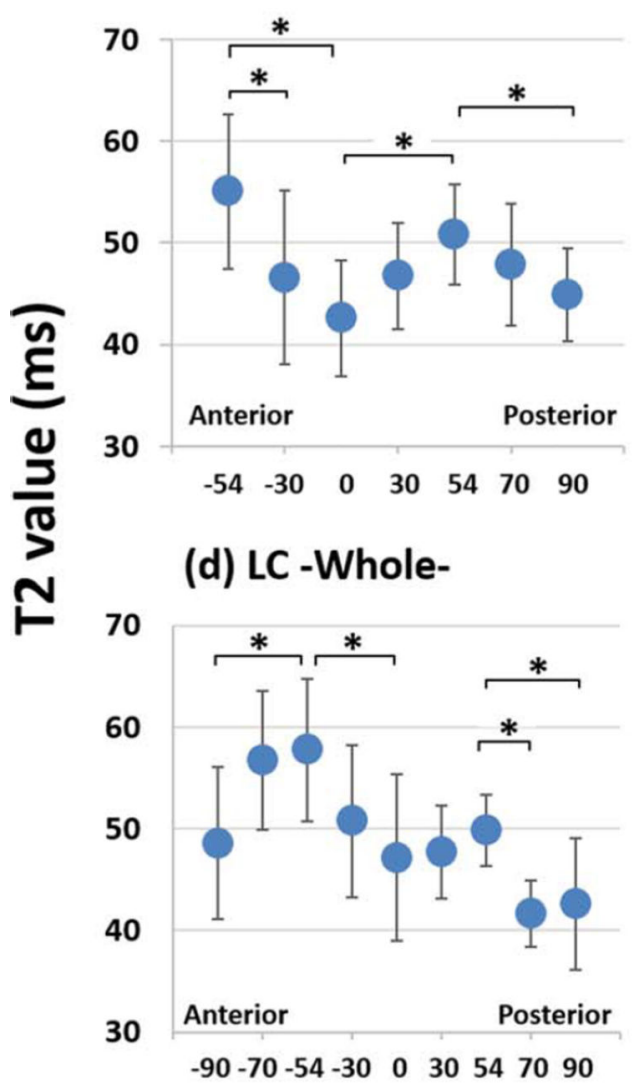

FIGURE 8. (a) MC -Whole-

(d) LC -Whole-

(b) MC -Deep-

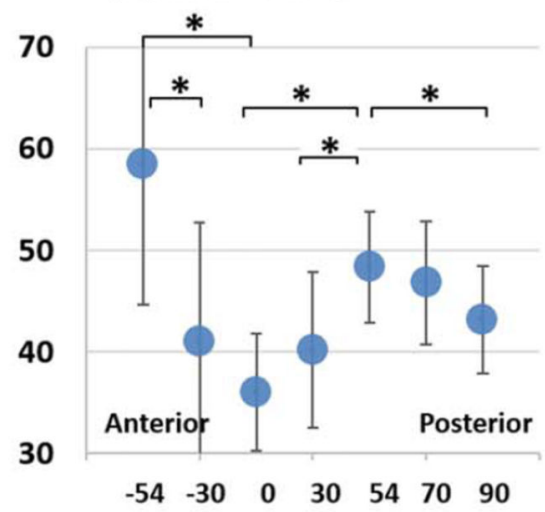

(e) LC -Deep-

70

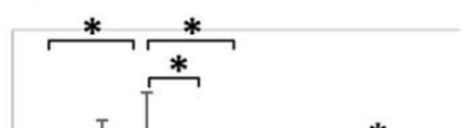

60

60

50

40

30

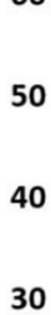

30

\section{Angular orientation to $\mathrm{B}_{0}$ (degrees) $\quad *_{p}<0.05$}

Comparison of $T_{2}$ values between magic angle and other representative angles with respect to each layer. MC medial condyle, T trochlea, LC lateral condyle.

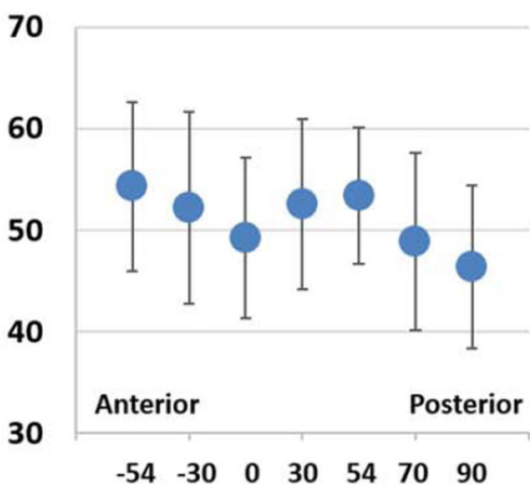

(f) LC -Superficial-

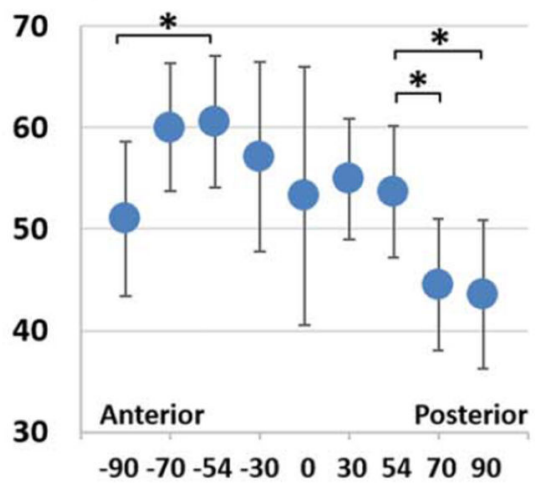




\section{(a) Subject 5 slice $14(T+6)$}

(b) Subject 11 slice 19 (LC-3)
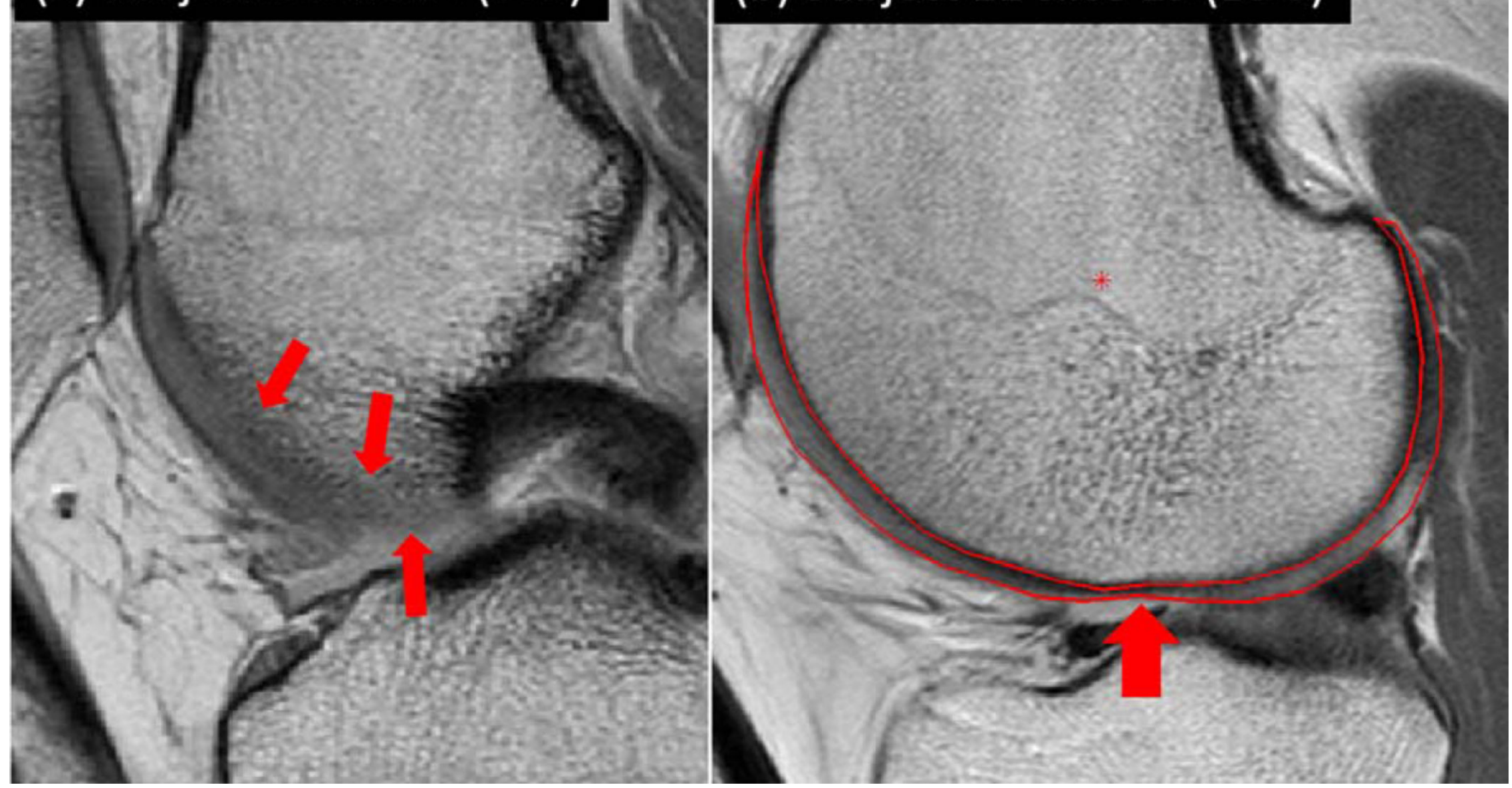

FIGURE 9.

$T_{2}$ mapping images where manual cartilage extraction was difficult: (a) a transitional location between trochlea and lateral condyle with the worst inter- and intrarater reliability, and (b) lateral condyle with the lateral femoral notch. T, trochlea; LC, lateral condyle. Phrases shown in parentheses indicate the center of femoral compartment \pm distance (mm). 


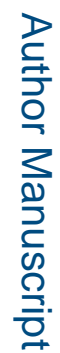

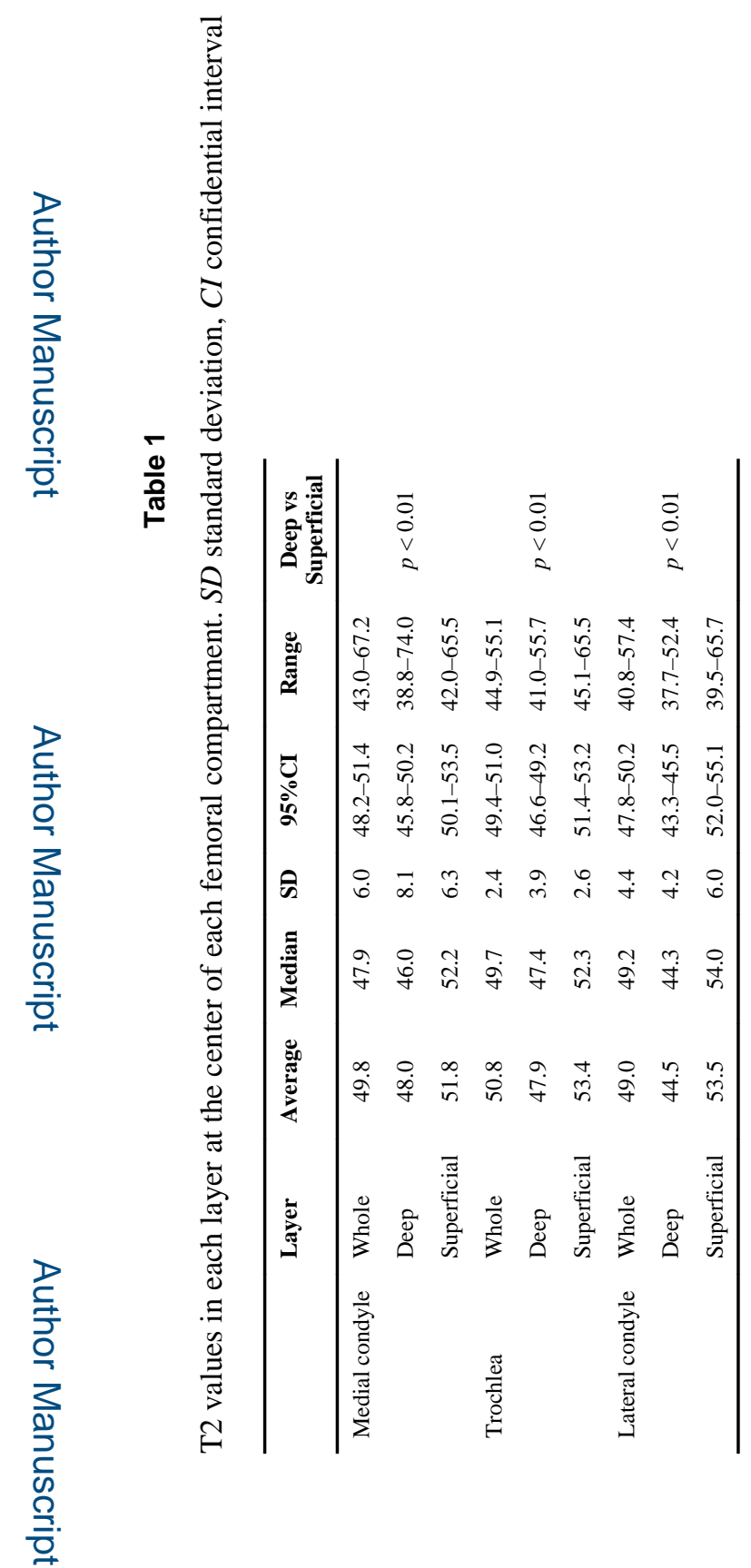

J Magn Reson Imaging. Author manuscript; available in PMC 2016 December 01. 